\title{
La cognoscibilidad del pasado pre-europeo. Problemas y propuestas
}

La compréhension du passé pré-européen. Problème et propositions

The problems and proposals for a definition of comprehensibility of the preeuropean past

\section{Peter Kaulicke}

\section{(2) OpenEdition}

Journals

\section{Edición electrónica}

URL: http://journals.openedition.org/bifea/4838

DOI: $10.4000 /$ bifea.4838

ISSN: 2076-5827

\section{Editor}

Institut Français d'Études Andines

\section{Edición impresa}

Fecha de publicación: 1 diciembre 2005

Paginación: 289-297

ISSN: 0303-7495

\section{Referencia electrónica}

Peter Kaulicke, «La cognoscibilidad del pasado pre-europeo. Problemas y propuestas », Bulletin de I'Institut français d'études andines [En línea], 34 (3) | 2005, Publicado el 08 diciembre 2005, consultado el 15 diciembre 2020. URL : http://journals.openedition.org/bifea/4838; DOI : https://doi.org/10.4000/ bifea.4838

\section{(c) (i) $\odot$}

Les contenus du Bulletin de l'Institut français d'études andines sont mis à disposition selon les termes de la licence Creative Commons Attribution - Pas d'Utilisation Commerciale - Pas de Modification 4.0 International. 


\title{
La cognoscibilidad del pasado pre-europeo. Problemas y propuestas
}

\author{
Peter Kaulicke*
}

\begin{abstract}
Resumen
¿Cómo se puede alcanzar un conocimiento del pasado de los pueblos que vivían en América del Sur antes de la llegada de los europeos? ¿Existen nociones de conciencia histórica pese a la ausencia de escritura o se trata de una mitificación basada en las memorias actuales o en invenciones de pasados guiados por intereses políticos? En el siguiente artículo se trata de buscar respuestas a estas preguntas al discutir enfoques poco conocidos del inicio de los estudios respectivos (Uhle) y modernos en áreas tradicionalmente poco consideradas como espacios históricos (Amazonía). Estos enfoques postulan una aceptación de historicidad que se puede obtener por medio de trabajos interdisciplinarios, internacionales y comparativos dentro de conceptos más científicos que políticos. Como ejemplo se presenta las experiencias obtenidas por la realización y publicación de dos simposios internacionales organizados por el autor en la Pontificia Universidad Católica. Estas experiencias apuntan hacia la necesidad de discusiones sostenidas sobre aportes empíricos y teóricos en foros que permitan el intercambio libre con el fin de avanzar hacia una aceptación del pasado pre-europeo dentro de la complejidad y dinámica que le caracteriza en vez de aceptar nacionalismos que enfatizan lo «exótico» y esencialmente inalterado de pasados que legitiman la presencia de los estados nacionales modernos.
\end{abstract}

Palabras clave - cognoscibilidad, pasado pre-europeo, América del Sur, historia pre-europea, enfoques interdisciplinarios, perspectivas comparativas

\section{La compréhension du passé pré-européen. Problème et propositions}

\section{Résumé}

Comment peut-on parvenir á une connaissance du passé des peuples qui vivaient en Amérique du Sud avant l'arrivée des Européens ? Existait-il des notions de conscience historique malgré l'absence d'écriture ou s'agit-il d'une mystification basée sur les mémoires actuelles ou sur les inventions de passés guidées par des intérêts d'ordre politique ? Dans cet article, nous tentons d'apporter des réponses

Profesor principal de la Pontificia Universidad Católica del Perú, Departamento de Humanidades, Apto. 1761, Lima. E-mail: pkaulic@pucp.edu.pe 
à ces questions en présentant des points de vue peu connus, qui remontent aux premières études sur le sujet (Uhle) et à celles de l'époque moderne, dans des régions qui sont traditionnellement peu considérées comme des espaces historiques comme l'Amazonie. Ces points de vue renvoient à une acceptation de l'historicité qui est mise en évidence par des travaux interdisciplinaires, internationaux et comparatifs dans le cadre de concepts plus scientifiques que politiques. Nous présentons comme exemple les expériences de réalisation et de publication de deux symposiums internationaux organisés par l'auteur de cet article à la Pontificia Universidad Catolica du Pérou. Ces expériences prouvent qu'il est nécessaire d'engager le débat à partir d'apports empiriques et théoriques au sein de forums qui permettent un échange libre afin d'aller vers une acceptation complexe et dynamique du passé préeuropéen dans le cadre de la complexité et de la dynamique qui lui sont propres au lieu d'accepter les nationalismes qui mettent l'accent sur l'exotisme et sur ce qui perdurerait sans altération du passé et légitimerait en quelque sorte la présence des États nationaux modernes.

Mots clés - compréhension, passé pré-européen, Amérique du Sud, histoire pré-européenne, approche interdisciplinaire, perspectives comparatives

\title{
The problems and proposals for a definition of comprehensibility of the pre-european past
}

\begin{abstract}
How can we achieve knowledge of the past of peoples who lived in South America before the arrival of the Europeans? Did they possess notions of historical consciousness in spite of the absence of written sources or are we confined to myths based on present memories or inventions of a past guided by political interests? This paper aims at answers for these questions by discussing little known contributions from the beginning of scientific work in the Americas (Uhle) and modern approaches in areas traditionally considered as devoid of historical spaces (Amazon). These approaches are based on the acceptance of early historicity that can be obtained by applying interdisciplinary, international, and comparative studies within scientific guidelines instead of politics. This is exemplified by the presentation of the experiences from two international symposia Pontificia Universidad Católica. These experiences resulted in the necessity of sustained discussions on empirical and theoretical advances towards the acceptance of a complex and dynamic pre-European past that seem to represent this past better than nationalisms which are used to «exotize» essentially unaltered pasts in order to legitimize the presence of modern national states.
\end{abstract}

Key words-comprehensibility, Pre-European past, South America, Pre-European history, interdisciplinary approaches, comparative perspectives

Dos trabajos, uno presentado hace casi 90 años y otro reciente, servirán para iniciar la discusión del tema que está señalado en el título. El autor del primero es F. Max Uhle, el iniciador de la arqueología científica en una serie de países latinoamericanos. Su aporte no siempre está reconocido debidamente a causa de las orientaciones marcadamente nacionalistas en muchos de estos países, surgidas con posterioridad a sus intervenciones. A modo de ejemplo quisiera citar un párrafo de un libro reciente, en el cual se crea una dicotomía marcada entre Uhle y el fundador de la arqueología nacional en el Perú, Julio C. Tello: 
«Tello y Uhle son indudablemente dos pilares opuestos de la arqueología peruana. Tello representa el nacionalismo de una arqueología comprometida con el presente. Uhle es el investigador extranjero encerrado en un gabinete de trabajo, ignorando el presente, la identidad nacional y la conservación de los monumentos arqueológicos, actitud seguida hasta la fecha por todos los arqueólogos extranjeros que trabajan en el Perú. Más aún, creemos que son pilares distintos en la consecución de la arqueología como ciencia, aspecto que se hace notar después de la muerte de Tello». (Morales, 1993: 19)

Esta posición, compartida por muchos arqueólogos peruanos, quizá no siempre en esta forma algo extrema, no corresponde a lo que Uhle ha realizado en cinco países de América del Sur durante más de 40 años de su vida como científico (véase Kaulicke, 1998b, y en cuanto a la discusión entre las posiciones de Tello y Uhle, Kaulicke, 1998a). Este tema se refleja bien en un trabajo de Uhle que está muy poco conocido. Se trata de una ponencia presentada en el Segundo Congreso Panamericano en Washington en 1915-1916, publicada bajo el título Conveniencia de dictar una ley uniforme en los países americanos para proteger y estimular el estudio y recolección de material arqueológico y antropológico (Uhle, 1917, reeditado en Kaulicke, 1998: 301-335). Es la sorprendente propuesta de una ley panamericana, la que no prosperó como no se ha logrado algo parecido hasta la actualidad. Su ferviente defensa del patrimonio prehispánico (no solamente peruano) contradice en forma rotunda la citada opinión ligera expresada por el arqueólogo peruano. Pero lo que interesa no es esta propuesta de ley, que Uhle llama jus historiae antiquae americanae (derecho de la historia antigua americana), sino su fundamentación. En sus palabras, este derecho no sirve

«[...] para subyugar [a las naciones americanas], no para imponerles una voluntad superior localizada en una u otra parte, sino para fortalecer a cada una de ellas individualmente, facilitándoles que se armen con las armas de la historia contra cualquier pretendiente interior o externo, i mutuamente contra cualquiera que viniera a probar que no ocupan en su totalidad el continente con título a justicia». (Uhle, 1917: 403)

Es, por tanto, la historia antigua americana que le da la fuerza legal a este proyecto. Esta historia, en el concepto de Uhle, se divide en historia convencional, iniciándose con la llegada de los europeos, y la pre-europea, la cual, según Uhle,

«[...] se ha de preparar por medio de la arqueolojia con sus ciencias hermanas la antropolojia, i, aunque en sentido menor, por la pérdida de la mayor parte de las lenguas antiguas, la lingüística». (Uhle, 1917: 387)

La arqueología no solo sirve para documentar estos materiales,

«[...] sino usa [los materiales] al mismo tiempo para la reconstrucción del desarrollo de las civilizaciones pasadas una de otra, de los factores que han contribuido a formarlas, de sus migraciones, paulatina estencion, de las causas que sirvieron a producirlas i despues a perderlas, entonces para la reconstrucción de su historia». (Uhle, 1917: 387)

Se trata de una especie de

«[...] filosofía que debe hacer comprender las fuentes de que el estado ha nacido i cuya multiplicación i desarrollo lójico tiene que dar como un resultado forzoso todo su desenvolvimiento hasta su fin». (Uhle, 1917: 386)

Insiste en la necesidad de su definición ante la indiferencia e incomprensión de esta necesidad:

«Los estados americanos son jóvenes, en los campos más variados de la vida no se han despertado todavía al entendimiento de su deber, disipan las fuerzas de su juventud sin pensar en las necesidades que tendrán en el tiempo de su madurez. Ahora la historia les parece de poca importancia, gozan su vida como si nunca hubiese de venir el tiempo en que necesitarán las fuerzas sacadas de las enseñanzas del pasado para mantenerse derechos. Sin embargo este tiempo ha de venir a ellos como ha venido para todos los otros estados modernos del mundo. Vendrá por eso el tiempo en que por necesidad se habrán de acordar de las fuentes de la historia precolombiana del suelo que ocupan para 
encontrarlas quizá después exhaustas por la indiferencia punible de los siglos pasados. Entonces les faltarán las raíces profundas echadas en el suelo por medio de la historia, i como plantas acuáticas sin raíces parecerán expuestos a ser desalojados por el viento en el océano del continente que nunca habían hecho suyo penetrando en su pasado». (Uhle, 1917: 393)

La comprensión de la historia antigua, para Uhle, no es una cuestión de moral, sino de sensatez política, y es un problema de identidad mejor resuelto por los estados europeos que gozan de conciencias históricas, basadas en historias largas. La supuesta inexistencia en el caso americano es una ilusión que se debe al afán intencional de rechazar o de ignorar historias diferentes de profundidades comparables a las europeas. Pero Uhle también se opone a los nacionalismos en constatar que:

«[l]a historia de ninguna parte del mundo se ha desarrollado sin enlazamiento continuo con las rejiones vecinas. Ningún país por eso puede llenar la reconstrucción de su propia historia moderna o antigua sin continua referencia a condiciones o acontecimiento que existen o han pasado en rejiones vecinas. De esto se deriva cierto derecho de cada país aislado a que también la historia del vecino se estudie hasta poder servir como ayuda en la explicación de su propio desarrollo». (Uhle,1917: 399)

Esta idea proviene de la experiencia europea también, pero, para Uhle, es aplicable a las Américas:

«Por su configuración geográfica especial el continente americano formó, más que otras grandes partes de nuestro planeta, una unidad también con respecto al orijen de su población antigua i al desarrollo de su civilizaciones... Los tipos de la antigua población presentan una enorme variedad en el norte i sur, i casi en todas partes simultáneamente, cuya descendencia i orijen solo se puede aclarar con el concurso científico de todos los países». (Uhle, 1971: 400)

Lo que Uhle plantea, en fin, es un enfoque comparativo y «holístico», interdisciplinario, internacional y esencialmente histórico, que debería extenderse a todo el continente cuya justificación no solo se basa en lo científico, sino también en lo político. Queda claro, sin embargo, que esto no implica que lo político condicione lo científico, sino al revés, lo científico debería condicionar lo político.

El segundo trabajo es fruto de un evento internacional con el título Comparative Arawakan histories. Rethinking Language Family and Culture Area in Amazonia de 2002, editado por Jonathan Hill y Fernando Santos-Granero (Hill \& Santos-Granero, 2002). Como ya indica el título el enfoque es interdisciplinario (historia, etnología, arqueología y lingüística), dedicado a un área vasta que abarca buena parte del continente sudamericano con la excepción del cono sur. Se trata además de la presencia de grupos cuya capacidad de generar historia se suele ignorar, cuya mera existencia aún se pone en duda, pese a la presencia muy prolongada del hombre en esta área y las evidencias de sociedades complejas con anterioridad a los europeos existentes aún en los siglos XVI y XVII (véase McEwan et al., 2001). En el trabajo citado se discuten diferentes ángulos como la relación entre lenguas, culturas e historias locales, jerarquía, diáspora e identidades nuevas, así como poder, cultismo y paisajes sagrados. Problemas controvertidos y poco populares en la arqueología como migraciones, correlación entre lenguas y grupos culturales, profundidad temporal de historia orales, etnogénesis y lingüística comparada no solo se discuten sino se aceptan como paradigmas. Si bien los resultados aún son algo tentativos en muchos aspectos, la utilidad del enfoque en sí me parece demostrada (véase también Whitehead, 2003).

Estos dos ejemplos demuestran que las ideas de entender el pasado pre-europeo esencialmente como historia no solo son de larga data, sino también de renovado interés en los últimos años. Estas construcciones de historia o historias solo tienen sentido si se las aplica en un sentido comparativo y, si es posible, dentro de una perspectiva globalizante. Esto significa que no deberían construirse islas caracterizadas por la presencia de historias complejas en un mar de una nebulosidad cuasi ahistórica o, como se solía expresar por centros productivos e innovadores y 
periferias pasivas o esencialmente inalteradas. «Historia» es tanto una definición de temporalidad como también concepto de la misma, una conciencia del pasado como el pasado en su propio derecho sin su necesaria presencia en el presente.

Quisiera ejemplificar esta idea con las experiencias ganadas en dos simposios organizados en la Pontificia Universidad Católica del Perú en Lima, en el 2000 (Kaulicke \& Isbell, 2000; 2001) y en el 2002 (Kaulicke et al., 2002; 2003; 2004). El primero llevó el título Huari y Tiwanaku: modelos vs. evidencias y contó con la participación masiva de reconocidos expertos (en total 66) en el tema, provenientes de 10 países, casi todos arqueólogos y un lingüista. Este tema «internacional» por regla no se trata en conjunto, menos en una perspectiva comparativa (Huari vs. Tiwanaku) por lo cual las ideas y conceptos vertidos, relacionados con el «fenómeno» Huari, difieren marcadamente de aquellos propagados sobre Tiwanaku ya que trabajos comparativos, tanto internos como entre ambas áreas, y otras influenciadas, escasean y a menudo carecen de la debida precisión metodológica o teórica. La definición de sistemas políticos complejos como «imperios», «reinos» o sus conversiones semánticas en términos «andinos» implica la necesidad de historizar estos fenómenos en una especificidad que permita comparaciones con fenómenos análogos en otras partes del mundo, donde éstos se conocen además por fuentes de otro tipo que las arqueológicas. El afán de reducirlos y, a la vez estereotiparlos y politizarlos, a una especie de gramática estilística (estilos emblemáticos dominantes vs. estilos locales o regionales largamente indefinidos o aún no aceptados como tales) o interpretaciones iconográficas (dentro de una lógica prestada de la historia de arte europea, pero sin los textos «explicativos») no constituye una alternativa viable. Se debe introducir otros conceptos como identidad social para poder identificar sus actores dentro de su concreto espacio social tanto como individuos, grupos o etnias (etnicidad) y la dinámica de sus transformaciones debido a negociaciones con otras identidades en acciones de diferentes grados de intensidad, así como también aspectos relacionados con la lingüística histórica. Estos contactos implican tanto transformación de nivel provincial como de los centros. Pero las provincias constituyen entidades históricas diferentes, con sus historias propias, por lo cual no deberían reducirse a una especie de pantalla para manifestaciones del poder central. En este sentido, las vigentes nociones generales y largamente opuestas de lo que se considera imperios, no se sustentan, en primer lugar, en evidencias concretas, sino en la transferencia de un modelo idealizado del «militarismo» inca de un agresivo estado expansivo con pleno dominio territorial gracias a un rígido sistema administrativo muy en boga en la década de los setenta durante el régimen militar en el Perú. Este enfoque parece poco prometedor ya que las evidencias señalan una complejidad mucho mayor con la presencia de sistemas políticos de grados diferentes de complejidad que corresponden a diferentes tradiciones «urbanas», centros y territorios cambiantes, profusión de estilos coexistentes e interpenetración de rasgos culturales que desafían el trazo de fronteras rígidas (véase Kaulicke, 2000; 2001) en un panorama complejo de diferentes historias con dinámica propia al lado de elementos más «internacionales» que no solamente se limitan a impulsos de la capital Huari. Esta capital tampoco es el único «centro del centro» sino comparte su papel con otro complejo grande, Conchopata (Isbell, 2000).

Tiwanaku, el otro centro de relevancia «internacional» y para muchos otro imperio de características diferentes con relación a los contactos con sus colonias (véase Kaulicke \& Isbell, 2001; Stanish, 2003; Janusek, 2004), fue una ciudad con una historia muy larga cuya complejidad se está revelando gracias a las intensivas excavaciones realizadas en los últimos años (Janusek, 2004). Tampoco Tiwanaku fue una «capital» única, sino comparte este papel con Lukurmata. Conviene detenerse en las caracterizaciones que Janusek (2004: 278-279) atribuye a las ciudades (cities) de Tiwanaku y Lukurmata. Ambas emergen gracias a la formación de redes jerárquicas de asentamientos, la construcción de complejos monumentales, un gradiente ascendente de estatus social y orientaciones definidas en cuanto a su ubicación y fenómenos naturales significativos. La vida residencial involucraba la creación, distribución y el uso de bienes valorizadas en un estilo fácilmente reconocible, tanto para el uso diario como en fiestas. Este contorno físico y social proporcionaba una cultura estatal adoptada, adaptada, 
reproducida y valorada por todos, resultando en un sentido profundo de identidad común y una experiencia de la coherencia comunitaria. Pero también era social e ideológicamente diverso, con énfasis en el poder local y la autonomía, lo que convertía los grupos residenciales en microcomunidades e incluía enclavados con afiliaciones con regiones distantes. Tiwanaku, por tanto, era parcialmente un centro cosmopolita albergando grupos semiautónomos. Además, tanto Tiwanaku como Lukurmata, eran símbolos físicos creados y modificados de acuerdo a principios cósmicos en paisajes sagrados, así como lugares de peregrinaje y convergencia ceremonial, y, como tal, una especie de ancla de unidad política global, afiliación religiosa e identidad cultural. Los diferentes lugares ceremoniales monumentales fueron construidos en diferentes etapas como centros de cultos distintos y de actividades rituales específicos, quizá fundados por grupos distintos de élite, castas sacerdotales o dinastías. Sus significados varían en el tiempo lo que convierte a Tiwanaku en un fenómeno religioso sincrético caracterizado por una significativa diversidad ritual y por el cambio. Ambos centros también eran tanto centros de valores de élite y de hegemonías culturales como lugares donde grupos con estatus diversificado, trasfondos, redes socioeconómicas, afiliaciones religiosas y parentescos residían y se visitaban. Estos grupos mantenían prácticas rituales y domésticas, patrones distintos de cultura material, cultos a ancestros locales e identidades duraderas.

Estas perspectivas corresponden a políticas arcaicas en un nivel global y, por lo tanto, no convierten Tiwanaku en una excepción andina cuyo funcionamiento y significado estén caracterizados por lógicas marcadamente distintas que obliguen a explicaciones sui generis.

Dentro de esta perspectiva no puede sorprender que toda el área circumtiticaca, con el centro de Tiwanaku, sea un área sumamente compleja como una especie de «mediterráneo» andino con muchas de las implicancias que sugiere esta analogía. Es, asimismo, un paisaje cultural y sagrado o mejor dicho una secuencia de estos mismos cuya aceptación como «centro del mundo» parece haber sido «multinacional» y «multitemporal» (véase arriba). Estos conceptos tampoco son netamente andinos, y por tanto, solo comprensibles para «andinos», sino presentes en el mundo entero, por lo cual sus definiciones también deberían servir para convertirlos en conceptos comparables.

Esta complejidad está especificada en el reciente hallazgo espectacular en la isla Pariti en el lago Titicaca (Korpisaari \& Pärssinen, 2005). Se trata de un depósito ritual de un gran número de vasijas de alta calidad, pertenecientes al estilo Tiwanaku (por fechados radiocarbónicos entre 800 y 1000 d. C.). Muchas de estas vasijas son vasos retratos de un «realismo» poco habitual en lo que se conocía de este estilo, que reflejan no solamente una enorme variedad en los rasgos físicos sino también en tocados y vestimenta que parecen reflejar diferentes conceptos de identidad y etnicidad. Me parece que estos rasgos no solamente señalan la probable presencia de diferentes etnias sino también contactos, aún poco claras, con conceptos estilísticos (ży de identidad?) mochica (costa norte del Perú) (véase Donnan, 2004).

Rasgos respectivos aparecen aún en regiones mucho más distantes como San Pedro de Atacama (Berenguer, 1986 y Kaulicke, 2001: 509, 518-519). San Pedro de Atacama (Llagostera, 2004) es otro centro, pero en este caso poco espectacular en dimensiones y complejidad interior, cuya importancia reside en la presencia de una multitud de estilos locales, regionales y de otros sorprendentemente distantes que involucran el área de Tiwanaku y el NO de Argentina (La Aguada).

Esta complejidad «internacional», la marcada dinámica en espacio y tiempo, identidades múltiples en diferentes niveles sociales y étnicos, rituales de gradación local, regional y suprarregional, no está reflejada debidamente en el «modelo militar» que se suele aplicar al «fenómeno» Huari, por lo cual es preciso diseñar modelos más apropiados en un ambiente caracterizado por complejidades de gradaciones diferentes con competencias más abiertas, nucleaciones importantes, estilos emblemáticos, sistemas diversos de comunicación, etc.

En el evento mencionado estos puntos fueron reconocidos en parte, pero pueden significar solo un punto de partida en el largo camino de una comprensión apropiada de los temas señalados. 
El segundo evento citado, con el título Identidad y transformación en el Tawantinsuyu y en los Andes coloniales. Perspectivas arqueológicas y etnohistóricas, puede entenderse como una extensión de la problemática anterior. Como el título indica, consistió en un diálogo entre etnohistoriadores y arqueólogos de diferentes nacionalidades, en el que participaron 76 especialistas de 14 países (en su mayoría etnohistoriadores y arqueólogos). Este diálogo, como el del tema Huari-Tiwanaku, no fue fácil tanto por conceptos de lo «incaico» que han tomado facetas variadas en los diferentes países donde se lo estudia como también por una cierta división entre historiadores y arqueólogos que usan terminologías compartidas que han adquirido significados diferentes en ambas disciplinas. Es, también, notable que muchos historiadores en su faceta de etnohistoriadores tienden a orientarse hacia la antropología o más concretamente a la etnografía en vez de la arqueología, en la cual, asimismo muchos investigadores tratan de buscar material etnográfico para la elaboración de sus enfoques teóricos. Esta tendencia es cuestionable ya que presume que subsisten elementos inalterados aplicables a ambas disciplinas. Es, sin embargo, obvio que una fusión de enfoques, el etnohistórico y el arqueológico, es inevitable para llegar a «rehistorizar» los incas en vez de aceptar idealizaciones políticas como cuasi hechos históricos. Como quedó claro, a los incas no se los entiende desde una exclusiva perspectiva «cuzco-céntrica» sin tomar en cuenta las complicadas negociaciones políticas y de identidades en procesos que afectan a ambos lados. Estos procesos, evidentemente, no terminan con la llegada de los europeos, sino crean nuevas negociaciones con estos últimos. Gracias a las tempranas fuentes escritas, los incas pueden ser estudiados mejor en vez de los conceptos del pasado, concentrados en mecanismos de memoria cultural, en particular con relación a Tiwanaku (cf. Kaulicke, 2004a). Por regla, se acepta que los incas emulaban la cultura material Tiwanaku (y/o Wari) como una revinculación con el pasado gracias a la iniciativa del Inca Pachacuti. Esta repetición algo irreflexiva de las fuentes escritas y un supuesto dark age con anterioridad a los incas que ellos terminan en su función de héroes culturales no necesariamente se encuentra confirmado por la arqueología. Hay demasiados «préstamos», en su mayoría relacionados con el ámbito de élite y de aspectos rituales que debería corresponder a otra lógica, la de un conocimiento mucho más preciso del papel de Tiwanaku y del «centro del mundo», el Lago Titicaca. Además de ello se comienzan a acumular datos de la presencia de sitios tiwanaku en la zona del Cuzco, el propio Cuzco incluido; la presencia de sitios grandes y complejos pertenecientes a Huari no está puesta en duda. Con ello no quiero subscribir la veracidad de la crónica de Montesinos de 1644 (véase Hiltunen, 1999; Hiltunen \& MacEwan, 2004). El periodo Intermedio Tardío, el tiempo entre el colapso de Tiwanaku (1150 d. C.) y la etnogénesis de los incas (antes de 1400 d. C.) reviste una complejidad política y étnica que no se puede reducir a una simple ruptura que puede haberse dado más por severas crisis ambientales que por razones de colapso interno y lo que Janusek (2004: 273) llama revolución cultural (1000-1200 d. C.) ya que está seguido por un nuevo proceso de complejización de élites, entre ellas los incas. Es poco probable que una compleja memoria cultural con tanto arraigo y profundidad temporal como la de Tiwanaku pueda ser eliminada totalmente en el lapso de tres a cuatro generaciones pese a cambios radicales en la cultura material (para una discusión más exhaustiva véase Kaulicke, 2004b).

Sin el afán de discutir en detalle la problemática relacionada a los incas y al Tawantinsuyu cuyos resultados se presentarán en breve (véase arriba) no quiero dejar de mencionar que este último está ahora tratado en perspectiva comparada con los imperios tempranos de otras partes del mundo (Alcock et al., 2001, Sinopoli, 2001). En esta perspectiva el imperio inca cabe muy bien en las definiciones básicas por lo cual no hay razón para buscar excepciones que lo separen como algo esencialmente distinto. Su lógica de funcionamiento y de expansión reviste, sin embargo, características propias que distan mucho de ser copias de las formaciones complejas como Huari y Tiwanaku.

Para terminar, queda por subrayar nuevamente que el papel de la arqueología no se limita a la construcción de secuencias basadas en materialidades eclécticas, traducidas luego en formaciones políticas complejas cuya sustentación proviene de reflexiones poco sistemáticas basadas en la lectura de textos tempranos. Precisa, en cambio, un reconocimiento de complejidades que no 
se restringen a ciclos de unidad y de desunión, de fuerza política y ausencia de ella, de dominio de etnias dominantes y estáticas frente a otras francamente indefinidas destinadas al olvido eterno, de nacionalismos arraigados en el origen de los tiempos y otros constructos basados en la negación de historia. Pienso que la posición de Uhle, en la esencia de sus ideas, en su llamado a la interdisciplinariedad y a la internacionalización de las historias, queda vigente como meta hacia una comprensión más apropiada del pasado de un continente cuya materialidad sumamente variada, sofisticada y de gran profundidad temporal en todas sus áreas llama a una sistematización más reflexiva y metodológicamente más refinada con enfoques más acordes a las exigencias científicas modernas de lo que es sujeta hasta la actualidad.

\section{Referencias citadas}

ALCOCK, S. E., D'ALTROY, T. E., MORRISON, K. D. \& SINOPOLI, C. M. (eds.), 2001 - Empires: perspectives from archeology and history, 523 p.; Cambridge: Cambridge University Press.

BERENGUER, J., 1986 - Relaciones iconográficas de larga distancia en los Andes. Nuevos ejemplos para un viejo problema. Boletín del Museo Chileno de Arte Precolombino, 2: 33-53; Santiago de Chile.

DONNAN, C. B., 2004 - Moche portraits from ancient Peru, 188 p.; Austin: University of Texas Press.

HILL, J. \& SANTOS-GRANERO, F. (eds.), 2002 - Comparative arawakan histories: rethinking language family and culture area in Amazonia, 340 p.; Illinois: University of Illinois Press.

HILTUNEN, J. J., 1999 - Ancient Kings of Peru. The Reliability of the Chronicle of Fernando de Montesinos, Correlating the Dynasty Lists with Current Prehistoric Periodization in the Andes, 511 p.; Helsinki: Suomen Historiallinen Suera.

HILTUNEN, J. J. \& McEWAN, G. F., 2004 - Knowing the Inca Past. In: Andean Archaeology (Silverman, H., ed.): 237-254; Malden: Blackwell Publishing.

ISBELL, W. H., 2000 - Repensando el Horizonte Medio: el caso de Conchopata, Ayacucho, Peru. In: Huari y Tiwanaku: Modelos vs. Evidencias. Primera Parte (Kaulicke, P. \& Isbell, W. H., eds.): 9-68; Lima: Fondo Editorial Pontificia Universidad Católica del Perú. Boletín de Arqueología PUCP 4.

JANUSEK, J. W., 2004 - Identity and Power in the Ancient Andes. Tiwanaku Cities Through Time, 288 p.; New York/London: Routledge.

KAULICKE, P., 1998a - Julio C. Tello vs. Max Uhle en la emergencia de la arqueología peruana y sus consecuencias. In: Max Uhle y el Perú Antiguo (Kaulicke, P., ed.): 69-82; Lima: Fondo Editorial Pontificia Universidad Católica del Perú.

KAULICKE, P. (ed.), 1998b - Max Uhle y el Perú Antiguo, 363 p.; Lima: Fondo Editorial Pontificia Universidad Católica del Perú.

KAULICKE, P., 2000 - La sombra de Pachacamac: Huari en la Costa Central. In: Huari y Tiwanaku: Modelos vs. Evidencias. Primera Parte (Kaulicke, P. \& Isbell, W. H., eds.): 313-358; Lima: Fondo Editorial Pontificia Universidad Católica del Perú. Boletín de Arqueología PUCP 4.

KAULICKE, P., 2001 - Cronología, identidad, urbanismo y estado en los Andes Centrales y Surcentrales entre los siglos $\mathrm{V}$ a X d. C.: algunas reflexiones finales. In: Huari y Tiwanaku: Modelos vs. Evidencias. Segunda Parte (Kaulicke, P. \& Isbell, W. H., eds.): 481-529; Lima: Fondo Editorial Pontificia Universidad Católica del Perú. Boletín de Arqueología PUCP 5. 
KAULICKE, P., 2004a - Memoria historiografiada y memoria materializada. Problemas en la percepción del pasado andino preeuropeo. Estudios Atacameños, 26 (2003): 17-34; San Pedro de Atacama.

KAULICKE, P., 2004b - Identidad, etnicidad e imperios: Algunas reflexiones finales. In: Identidad y transformación en el Tawantinsuyu y en los Andes coloniales. Perspectivas arqueológicas y etnohistóricas. Tercera Parte (Kaulicke, Urton \& Farrington, eds.): 325-357; Lima: Fondo Editorial Pontificia Universidad Católica del Perú. Boletín de Arqueología PUCP 8.

KAULICKE, P. \& ISBELL, W. H. (eds.), 2000 - Huari y Tiwanaku: Modelos vs. Evidencias. Primera Parte, 651 p.; Lima: Fondo Editorial Pontificia Universidad Católica del Perú. Boletín de Arqueología PUCP 4.

KAULICKE, P. \& ISBELL, W. H. (eds.), 2001 - Huari y Tiwanaku: Modelos vs. Evidencias. Segunda Parte, 658 p.; Lima: Fondo Editorial Pontificia Universidad Católica del Perú. Boletín de Arqueología PUCP 5.

KAULICKE, P., URTON, G. \& FARRINGTON (eds.), 2002 - Identidad y transformación en el Tawantinsuyu y en los Andes coloniales. Perspectivas arqueológicas y etnohistóricas. Primera Parte, 304 p.; Lima: Fondo Editorial Pontificia Universidad Católica del Perú. Boletín de Arqueología PUCP 6.

KAULICKE, P., URTON, G. \& FARRINGTON (eds.), 2003 - Identidad y transformación en el Tawantinsuyu y en los Andes coloniales. Perspectivas arqueológicas y etnohistóricas. Segunda Parte, 435 p.; Lima: Fondo Editorial Pontificia Universidad Católica del Perú. Boletín de Arqueología PUCP 7.

KAULICKE, P., URTON, G. \& FARRINGTON (eds.), 2004 - Identidad y transformación en el Tawantinsuyu y en los Andes coloniales. Perspectivas arqueológicas y etnohistóricas. Segunda Parte, 462 p.; Lima: Fondo Editorial Pontificia Universidad Católica del Perú. Boletín de Arqueología PUCP 8.

KORPISAARI, A. \& PÄRSSINEN, M., (eds.), 2005 - Pariti: Isla, misterio y poder. El tesoro cerámico de la cultura Tiwanaku, 80 p.; La Paz.

LLAGOSTERA, A., 2004 - Los antiguos habitantes del Salar de Atacama. Prehistoria atacameña, 213 p.; Santiago: Pehuén Editores.

McEWAN, C., BARRETO, C. \& NEVES, E. (eds.), 2001 - Unknown Amazon, culture in nature in Ancient Brazil, 304 p.; London: British Museum Press.

MORALES, D., 1993 - Historia arqueológica del Perú (del Paleolítico al Imperio Inca), 670 p.; Lima: Milla Batres. Compendio Histórico del Perú, tomo I.

SINOPOLI, C. M., 2001 - Empires. In: Archaeology at the Millenium (Feinman, G. \& Price, T. D., eds.): 439-471; New York: Kluwe Academic-Plenum Publishers.

STANISH, C., 2003 - Ancient Titcaca: the evolution of complex society in Souhtern Peru and Northern Bolivia, 354 p.; Berkeley: University of California Press.

UHLE, M., 1917 - Conveniencia de dictar una ley uniforme en los países americanos para proteger y estimular el estudio y recolección de material arqueológico y antropológico. In: Proceedings of the Second Pan American Scoientific Congress, Washington, D.C., U.S.A., Monday, December 27, 1915 to Saturday, January 8, 1919, Section I, Anthropology, Vol. I: 386-407; Washington. Reeditado en Max Uhle y el Perú Antiguo (Kaulicke, P., ed.): 301-335; Lima: Fondo Editorial Pontificia Universidad Católica del Perú.

WHITEHEAD, N. L. (ed.), 2003 - Histories and Historicities in Amazonia. 236 p.; Lincoln: University of Nebraska Press. 